\title{
Composición de la dieta de Tyto alba Scopoli en hábitats de Calabozo, Venezuela
}

\author{
Diet composition of Tyto alba in habitats of Calabozo, Venezuela
}

\author{
Lilian Fuentes $^{1 *}$, Isabel Sequera ${ }^{1}$, Carmen Poleo $^{2}$ y Lisbeth Díaz ${ }^{1}$ \\ ${ }^{1}$ Universidad Centroccidental Lisandro Alvarado, Decanato de Agronomía. Departamento de Ciencias Biológicas y \\ Departamento de Ingeniería Agrícola. Cabudare, Estado Lara, Venezuela. \\ ${ }^{2}$ Estación Experimental Instituto de Investigaciones Agrícolas. Calabozo, Estado Guárico, Venezuela. \\ * Autor para correspondencia (lfuentes@ucla.edu.ve) \\ Recibido: 13/04/2015; Aceptado: 09/06/2015. \\ http://dx.doi.org/10.18004/investig.agrar.2015.junio.46-53
}

\section{RESUMEN}

Para determinar la dieta de la lechuza de campanario (Tyto alba Scopoli, 1769) fueron colectadas 592 egagrópilas desde Enero hasta Diciembre de 2010, en la Estación Experimental del Instituto Nacional de Investigaciones Agrícolas, Calabozo, estado Guárico, Venezuela. Adicionalmente, en el sitio se registró la precipitación mensual durante la época seca y de lluvia. En el laboratorio, cada una de las egagrópilas fue medida para determinar tamaño y peso. Posteriormente fueron procesadas en seco para estimar la cantidad total de presas consumidas, especies en algunos grupos, número promedio de presas/egagrópila y las relaciones entre: número de presas consumidas y peso de las egagrópilas; y número de presas y la precipitación. Los promedios de peso, longitud y ancho de las egagrópilas fueron: $3,6 \mathrm{~g} \pm$ $1,5,2,7 \mathrm{~cm} \pm 0,6$ y $1,8 \mathrm{~cm} \pm 0,4$, respectivamente. $\mathrm{La}$ dieta de $T$. alba incluyó 847 presas, las cuales estuvieron conformadas por roedores (Zygodontomys brevicauda, Sigmodon alstoni, Holochilus sciureus y Oligoryomys sp.) (99,30\%), aves (Cuculidae y Passeriformes) $(0,23 \%)$ y murciélagos (Mollosus sp.) (0,47\%). El promedio de individuos por egagrópila fue $1,5 \pm 0,4$. El peso de las egagrópilas fue proporcional a la cantidad de presas $(\mathrm{r}=$ 0,4377; $\mathrm{P}=0,000)$. El número de presas consumidas y la precipitación estuvieron relacionados de manera inversa $(\mathrm{r}$ $=-0,7304 ; \mathrm{P}=0,007)$, la composición de la dieta de la lechuza no varió en ambos periodos. T. alba mostró ser un cazador potencial de roedores, útil en control biológico de roedores en el cultivo de arroz en Venezuela.

Palabras clave: Holochilus sciureus, Mollosus, Oligoryzomys, Sigmodon alstoni, Zygodontomys brevicauda.

\begin{abstract}
In order to determine the diet of the Barn Owl (Tyto alba Scopoli, 1769), 592 pellets were collected from January to December 2010 at one month interval, at the National Institute of Agricultural research's experimental center, in Calabozo, Guárico State, Venezuela. In addition, precipitation records during the dry and rainy seasons were obtained at the field research site. In the laboratory, each pellet was measured to determine size and weight. Then, each pellet was dry-processed to estimate total amount of consumed prey, species in some groups, the average number of prays per pellet and the relationship between the number of consumed preys and pellet's weight and between number of preys and rainfall. Mean weight, length and width of pellets were: $3.6 \mathrm{~g} \pm 1.5,2.7$ $\mathrm{cm} \pm 0.6$ and $1.8 \mathrm{~cm} \pm 0.4$, respectively. The owl's diet included 847 preys, comprised by rodents (Zygodontomys brevicauda, Sigmodon alstoni, Holochilus sciureus and Oligoryomys sp.) (99.30 \%), birds (Cuculidae and Passeriformes) (0.23\%) and bats (Mollosus sp.) (0.47\%). The average number of individuals per pellet was $1.5 \pm$ 0.4 . Pellets weight was directly related to number of prey ( $\mathrm{r}=0.4377 ; \mathrm{P}=0.000)$. Number of consumed prey and rainfall were inversely related $(\mathrm{r}=-0.7304 ; \mathrm{P}=0.007)$, however, diet composition was not affected by either rainy or dry season. T. alba showed to be a potential hunter of rodent and it can be useful as a biological agent against invasive rodent species in rice fields of Venezuela.
\end{abstract}

Key words: Holochilus sciureus, Mollosus, Oligoryzomys, Sigmodon alstoni, Zygodontomys brevicauda. 


\section{INTRODUCCIÓN}

La lechuza de campanario (Tyto alba Scopoli, 1769) es un ave rapaz, nocturna, perteneciente al orden Strigiforme, familia Tytonidae y de amplia distribución mundial (Goodman y Thorstrom 1998). Está adaptada a zonas rurales y urbanas (González et al. 2004, Aliaga-Rossel y Tarifa 2005) porque utiliza diversos sitios para anidar, incluyendo sitios construidos por el hombre (iglesias y edificios abandonados) (Aliaga-Rossel y Tarifa 2005, Mohamed y Santhanakrishnan 2012).

Los hábitos de alimentación de esta ave en diferentes hábitats han sido ampliamente estudiados en Sur América y otros continentes, mediante la utilización de la regurgitación de restos de alimento que no aprovecha (huesos, pelos, plumas, quitina de invertebrados), conocidos como egagrópilas (ICI 1987). A partir de este material se conoce que la lechuza se alimenta básicamente de pequeños mamíferos (roedores) y ocasionalmente otros vertebrados y artrópodos (González et al. 1999, Poleo y Agüero 2000, Ramírez et al. 2000, Trejo y Ojeda 2002, Bonvicino y Bezerra 2003, Teta y Contreras 2003, González et al. 2004, Aliaga-Rossel y Tarifa 2005, Pardiñas et al. 2005, Delgado y Ramírez 2009, Fernández et al. 2009, Rocha et al. 2011, Mohamed y Santhanakrishnan 2012, Nadeem, et al. 2012, Kitowski 2013). Por lo tanto, T. alba ha sido considerada excelente controlador biológico de roedores que ocasionan daños tanto, en la agricultura como en la salud pública (Mohd 1990, Mohamad y Goh 1991, Poleo et al. 1998, Poleo y Agüero 2000, Utrera et al. 2000, Hafidzi y Mohd 2003, Bontzorlos et al. 2005, Vásquez et al. 2005, Shehab y Al Charabi 2006, Magrini y Facure 2008, Rocha et al. 2011).

En Venezuela la mayor parte de las investigaciones sobre la dieta de la lechuza de campanario están referidas a egagrópilas localizadas en nidos artificiales instalados en lotes de arroz del Sistema de Riego del Río Guárico (SRRG), sólo durante el período reproductivo (Poleo et al. 1998, Poleo y Agüero 2000, Fuentes et al. 2012). A excepción de Araujo y Molinari (1995) quienes evaluaron la dieta de $T$. alba en la zona de los Andes y encontraron roedores, insectos, musarañas, aves y murciélagos. Sin embargo, no se han realizado evaluaciones sobre la dieta de $T$. alba en sitios naturales de descanso (posaderos), por lo que en el presente estudio se evaluó la composición y cantidad de la dieta de $T$. alba, así como su posible relación con la precipitación en una zona productora de arroz en Calabozo, estado Guárico.

\section{MATERIALES Y MÉTODOS}

La evaluación se llevó a cabo en la Estación Experimental del Instituto Nacional de Investigaciones Agrícolas (INIA), localizada en el km 28 carretera Calabozo a San Fernando de Apure en el Sector Los Bancos de San Pedro, municipio Miranda estado Guárico, Venezuela $\left(8^{\circ}\right.$ $45^{\prime} \mathrm{N}$ y $67^{\circ} 32^{\prime} \mathrm{W}$ ) (Figura 1 ).

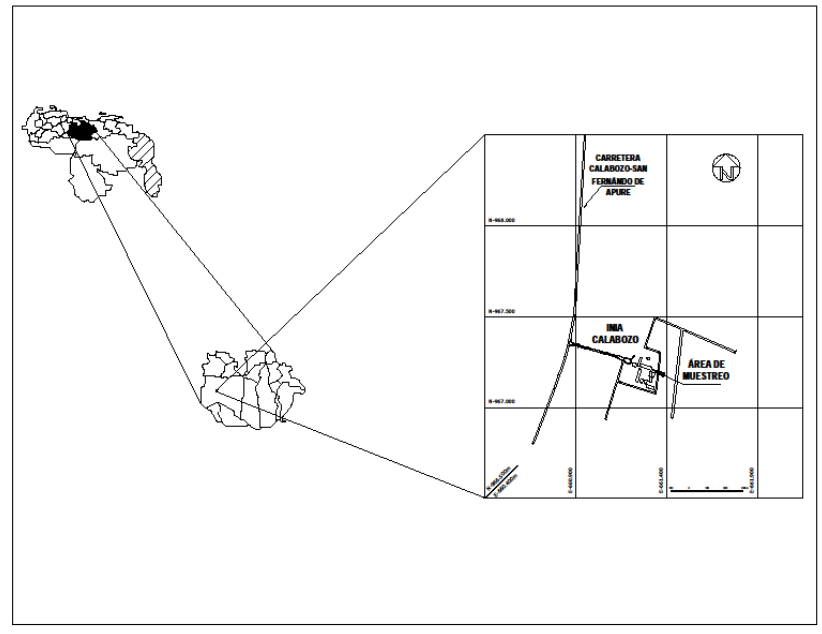

Figura 1. Ubicación relativa de los sitios de recolección de las egagrópilas de $T$. alba en la Estación Experimental del Instituto Nacional de Investigaciones Agrícolas (INIA) Calabozo, Venezuela.

La zona de muestreo pertenece a la subregión sabana de altiplanicie en los Llanos Centrales del Orinoco (Silva 2003). Por lo que constituye una topografía plana $(0,5$ $2 \%$ ) y los puntos de máxima elevación no superan 73 msnm. Se distingue una condición biestacional: período seco (diciembre-abril) y otro de lluvias (mayo-octubre). Según datos publicados por Lugo (2005), la pluviosidad, temperatura y humedad relativa promedio se encuentran alrededor de $1312,5 \mathrm{~mm}, 27^{\circ} \mathrm{C}$ y $74 \%$, respectivamente. Con radiación solar abundante durante todo el año y valores superiores a $400 \mathrm{cal} \mathrm{cm}^{-2}$ día $^{-1}$. La vegetación de la Estación Experimental estuvo constituida por árboles, algunos pastos naturales e introducidos y el cultivo de arroz (Oryza sativa L.). Entre las especies arbóreas destaca la presencia de: samán (Pithecellobium saman (Jacq.) Benth), caujaro (Cordia dentata Poir), caruto (Genipa americana L.), caro-caro (Enterolobium cyclocarpum (Jacq.) Griseb), caña fistola (Cassia moschata H.B.K), camoruco (Sterculia apetala (Jacq.) Karsten), guácimo (Guazuma ulmifolia Lam), masaguaro (Pithecellobium guachapele (H.B.K) Macbr). Los pastos que se distinguen son: lambedora (Leersia exandra Swartz), gamelotillo (Paspalum plicatulum Michx), 
canarana (Echinochloa pyramidalis (Land) Lyitch), pasto alemán (Echinochloa polystachya H.B.K), braquiarias (Brachiaria radicans Napper, B. arrecta Durt \& Sch, B. humidicola (Rendle), B. decumbens Stapf, B. brizanta (Hochst) STM), yaraguá (Hyparrhenia rufa (Nees) Stapf), guinea (Panicum maximun Jacq) (Poleo et al. 2011).

La recolección de las egagrópilas se realizó mensualmente durante el período enero a diciembre 2010, en ocho sitios de descanso de T. alba ubicados dentro y en los alrededores de las edificaciones del INIA. Las egagrópilas se colocaron en bolsas de papel previamente identificadas y trasladadas al laboratorio de Zoología Agrícola de la Universidad Centroccidental "Lisandro Alvarado" (UCLA). Cada una fue pesada en una balanza analítica y medida el largo y ancho con un vernier digital. Posteriormente fueron procesadas en seco, utilizando pinzas para extraer los restos de mandíbulas, cráneos de los animales encontrados. Aquellas egagrópilas que estaban compactadas fueron remojadas en agua durante 3 horas para realizar la separación.

Las mandíbulas y cráneos de los roedores capturados se compararon con muestras ideríificadas en la colección de Zoología Agrícola (UCLA). El material de aves y murciélagos fue enviado a especialistas de la Universidad Central de Venezuela (UCV) y de la Universidad de Los Andes (ULA), respectivamente. Se consideró como un individuo a los pares de mandíbulas de la misma especie y mandíbulas individuales.

La caracterización de las egagrópilas se realizó mediante una estadística descriptiva (promedios y desviación estándar) y la correlación de Pearson. Este último permitió determinar si estarían relacionados el peso de las egagrópilas y la cantidad de presas; la cantidad de individuos depredados y la precipitación de la zona. El programa utilizado fue Statistix versión 8.0.

\section{RESULTADOS Y DISCUSIÓN}

Caracterización de las egagrópilas de Tyto alba y la relación entre el peso de las egagrópilas y la cantidad de presas extraídas

Se procesaron 592 egagrópilas y se obtuvo un peso promedio $(3,6 \mathrm{~g} \pm 1,5)$ muy similar a los alcanzados por Soncini et al. (1985) y López (2012) en egagrópilas de $T$. alba de Argentina y Cuba, respectivamente. En las egagrópilas, se evidenció que el peso estuvo relacionado con la cantidad de individuos depredados $(\mathrm{r}=0,4377 ; \mathrm{P}=$
0,000). Resultado ya observado por Fuentes et al. (2012), quienes determinaron una relación entre ambas variables que permitió estimar la cantidad de presas a partir del peso de la egagrópila y por lo tanto, una forma rápida de evaluar la depredación cuando las egagrópilas no puedan ser procesadas de manera inmediata.

La longitud y ancho $(2,7 \mathrm{~cm} \pm 0,6$ y $1,8 \mathrm{~cm} \pm 0,4$, respectivamente), resultaron menores a las obtenidas por Soncini et al. (1985), Trejo y Ojeda (2002), Debus et al. (2004), López (2012), Senzota (2012). Las dimensiones de las egagrópilas dependen del tipo de alimento y del tamaño del rapaz (Soncini et al. 1985). En esas investigaciones, el espectro alimenticio de T. alba fue amplio y con presas de mayor tamaño a las registradas en esta evaluación.

El promedio de individuo por bolo fue 1,5 $\pm 0,4$. Este valor resultó consistente al obtenido por Soncini et al. (1985). Sin embargo, se han registrado entre uno a cinco individuos dependiendo de la disponibilidad y tamaño de la presa (Shehab y Al Charabi 2006, Hernández-Muñoz y Mancina 2011, López 2012).

La utilización de las egagrópilas como método para analizar la dieta de la lechuza de campanario es ampliamente conocida, sin embargo las medidas morfométricas generalmente no son analizadas en investigaciones sobre la dieta de la lechuza y sólo pocas evaluaciones dan algunos elementos que pudieran ser comparados (López 2012). El tamaño pudiera servir para diferenciar especies de Strigiformes que coexistan en un área determinada y el peso para obtener ecuaciones que permitan estimar (previa validación) de forma rápida la depredación, cuando las egagrópilas no puedan ser procesadas de manera inmediata.

\section{Especies y número de individuos depredados por Tyto alba}

Del análisis de las egagrópilas se determinó que los roedores fueron el grupo más representativo en la dieta de la lechuza, durante el período enero-diciembre 2010. Se estimó un total de 847 individuos, de los cuales 757 fueron roedores, pertenecientes a las especies: Zygodontomys brevicauda Allen y Capman (47,7\%), Sigmodon alstoni Thomas (26,0\%), Holochilus sciureus Wagner (10,2\%), Oligoryzomys Bangs (5,5\%), mientras que los roedores no identificados (mandíbulas sin molares) constituyeron 9,9\%. Adicionalmente, se encontraron dos cráneos de aves (Cuculidae y Passeriformes y cuatro de murciélagos (Mollosus sp) que representaron 0,7\% (Tabla 1). 
Tabla 1. Dieta de Tyto alba determinada mediante el número de egagrópilas colectadas en sitios de descanso de la Estación Experimental INIA Calabozo (Venezuela), durante el período enero-diciembre 2010.

\begin{tabular}{|c|c|c|c|c|c|c|c|c|}
\hline Mes & $\mathbf{N}$ & $\mathbf{Z}$ & $\mathbf{S}$ & $\mathbf{H}$ & O & NI & $*$ & Total \\
\hline 1 & 80 & 54 & 24 & 25 & 16 & 10 & 0 & 129 \\
\hline 2 & 101 & 76 & 38 & 10 & 11 & 7 & 1 & 143 \\
\hline 3 & 69 & 48 & 21 & 18 & 1 & 2 & 1 & 91 \\
\hline 4 & 60 & 52 & 24 & 7 & 4 & 13 & 2 & 102 \\
\hline 5 & 21 & 11 & 8 & 4 & 0 & 3 & 2 & 28 \\
\hline 6 & 21 & 22 & 4 & 3 & 1 & 7 & 0 & 37 \\
\hline 7 & 16 & 11 & 11 & 5 & 2 & 9 & 0 & 38 \\
\hline 8 & 9 & 14 & 4 & 0 & 1 & 0 & 0 & 19 \\
\hline 9 & 40 & 14 & 17 & 5 & 2 & 10 & 0 & 48 \\
\hline 10 & 21 & 11 & 15 & 2 & 1 & 2 & 0 & 31 \\
\hline 11 & 84 & 32 & 29 & 5 & 6 & 12 & 0 & 84 \\
\hline 12 & 70 & 59 & 25 & 2 & 2 & 9 & 0 & 97 \\
\hline Total & 592 & 404 & 220 & 86 & 47 & 84 & 6 & 847 \\
\hline
\end{tabular}

n: número de egagrópilas, (Z) Zygodontomys brevicauda, (S) Sigmodon alstoni, (H) Holochilus sciureus, (O) Oligoryzomys, NI: roedores no identificados * Otros individuos (1 Cuculidae, 1 Passeriformes y 4 Chirópteros).

Los hábitos alimentarios de la lechuza de campanario han sido objeto de estudio en varias partes del mundo, tanto en nidos como en posaderos (sitios de descanso), demostrando que esta ave se alimenta principalmente de pequeños roedores y ocasionalmente de otros vertebrados y artrópodos (Bellocq y Kravetz 1993, Delgado y Calderón 2007, Fernández et al. 2009, Hernández-Muñoz y Mancina 2011, Fuentes et al. 2012, López 2012). Las variaciones en la dieta están dadas, según el hábitat que rodea los nidos o sitios de descanso: zonas no intervenidas, cultivadas y/o urbanas.

Los posaderos donde se colectaron las muestras, estaban en las edificaciones de INIA Guárico y entre $500 \mathrm{~m}$ a 1 $\mathrm{km}$ existían 10 ha de arroz, el resto corresponde a zonas de potreros. Estas áreas se ubican dentro del área de acción de $T$. alba (2 km) (ICI 1987), lo cual podría explicar el alto porcentaje de $Z$. brevicauda, seguido de $S$. alstoni debido a que, ambas tienen preferencias de hábitat en cultivos mecanizados y pastizales (Linares 1998, Utrera et al. 2000). También, Z. brevicauda fue la especie más depredada por la lechuza de campanario localizadas en nidos artificiales y la más capturada en trampeos, seguido de $H$. sciureus, durante un año de muestreo sobre lotes de arroz de la Estación del INIA Calabozo (Poleo y Agüero 2000).

Z. brevicauda se mantiene activa durante toda la noche, mientras que la actividad de $S$. alstoni ocurre entre las 6:00 pm y 8:00 pm y luego entre las 5:00 am y 8:00 am (Vivas et al. 1986). Este comportamiento podría explicar porqué $T$. alba ejerció mayor depredación sobre la especie más disponible o activa en el área, posiblemente el depredador y la presa coincidan en sus actividades nocturnas. La mayor depredación sobre Z. brevicauda cobra importancia porque es reservorio del virus de la fiebre hemorrágica venezolana (VFHV), por lo tanto contribuye a mantener baja esta población (Fulhorst et al. 1999, Paredes 2012).

Aparte de Z. brevicauda, también fueron encontradas en menor proporción $H$. sciureus y Oligoryzomys y su ocurrencia podría ser explicada por la proximidad del cultivo de arroz, el cual es hábitat para estas especies (Poleo et al. 1998). De manera similar, las aves y murciélagos como parte de la dieta fueron poco relevantes, lo cual pudiera tener un carácter casual, hecho ya observado por Soncini et al. (1978), Araujo y Molinari 
(1995), Poleo y Agüero (2000), Debus et al. (2004), Delgado y Calderón (2007), Santos-Moreno y Alfaro 2009, Hernández-Muñoz y Mancina (2011), Rocha et al. (2011). No obstante, algunos han citado porcentajes importantes de depredación sobre estos vertebrados (Teta y Contreras 2003, González et al. 2004, López 2012).

Las diferencias en la dieta podrían relacionarse con la conducta oportunista de la lechuza, ante la disminución estacional en las poblaciones de pequeños mamíferos que dispara una respuesta facultativa hacia la abundancia de recursos tróficos secundarios (González et al. 2004, Fernández et al. 2009).

\section{Relación entre la cantidad de animales depredados y la precipitación}

Se encontró una relación negativa entre la cantidad de animales depredados y la precipitación $(\mathrm{r}=-0,7304 ; \mathrm{P}=$ 0,007), lo cual indica que hubo mayor cantidad de roedores consumidos durante los meses de menor precipitación (Figura 2).

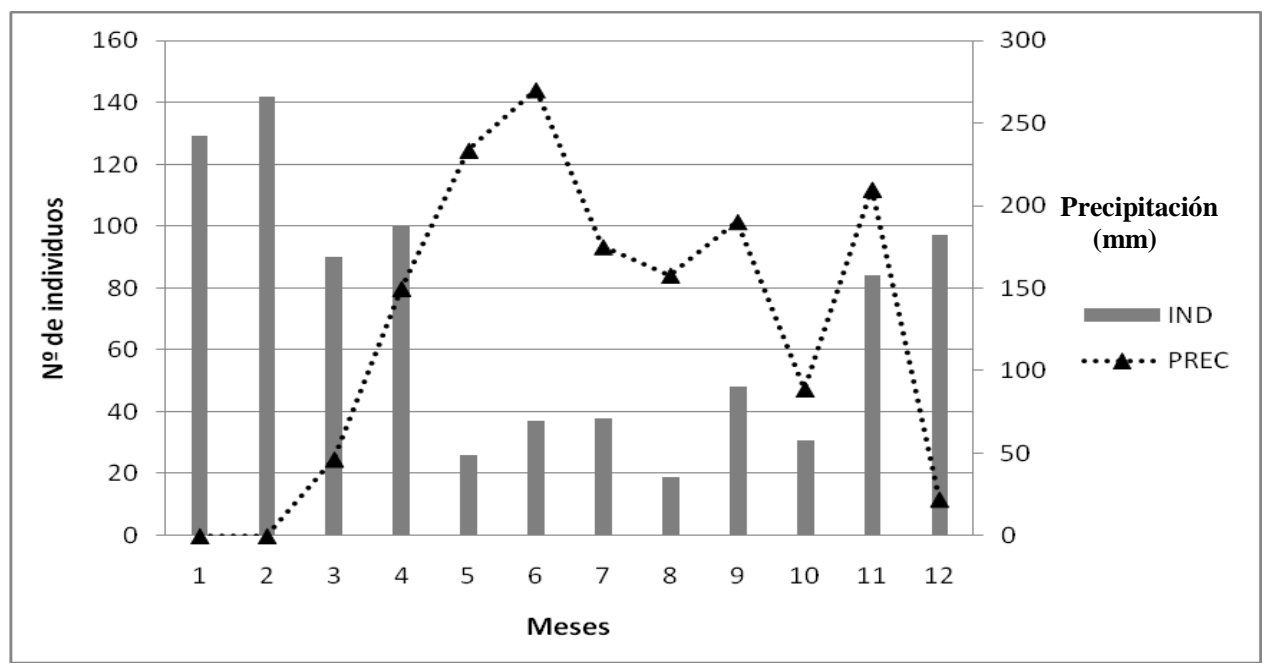

Figura 2. Distribución de la precipitación y la cantidad de animales depredados por Tyto alba en la Estación Experimental INIA-Calabozo (Venezuela), durante el período enero-diciembre 2010.

La precipitación puede tener un impacto negativo sobre el comportamiento de caza o la habilidad de percepción de la presa (Olsen y Olsen 1992, Rubolini et al. 2003). Durante la época de lluvia la lechuza realiza más esfuerzo para cazar porque la vegetación es más abundante, de hecho se han observado lechuzas cazando en las orillas de las carreteras para capturar roedores que van de un lado a otro de la vía (observación personal). Mientras que, en sequía la vegetación es escasa y las labores agronómicas como quema, limpieza de los bordes del cultivo y preparación de suelo causan la salida de los roedores de las madrigueras quedando más expuestos a la depredación.

Adicionalmente, la reproducción de la lechuza (septiembre-abril) (Poleo et al. 1998) se ha sincronizado con la época de mayor superficie de siembra de arroz en Venezuela (período de sequía) (MAT 2005-2007) y por lo tanto mayor incidencia de roedores en el cultivo. Esto le permite al ave rapaz capturar suficientes presas en menos tiempo, asegurando de esa manera el éxito reproductivo (Zuberogoitia 2000).

En esta evaluación se observó que durante todo el año $T$. alba consumió las mismas especies de roedores, las cuales son las que tradicionalmente se capturan en los muestreos que se realizan en la Estación del INIA (Mendoza 1990, 2006 y 2007). Los cambios sólo se observaron en la cantidad de presas. Aunque, en otros estudios se ha determinado variaciones estacionales de la dieta de esta ave atribuida a los cambios en la disponibilidad y comportamiento de los pequeños mamíferos en diferentes estaciones y años (Brown 1981, Campbell et al. 1987).

En general, las condiciones climáticas influencian la composición de la dieta debido a que pueden afectar tanto la actividad de las aves rapaces como la abundancia de la 
presa (Rubolini et al. 2003), condicionando así su potencial de depredación. En tal sentido, las investigaciones futuras deberían incluir la cuantificación del ave, dieta y área de acción, captura simultánea de pequeños mamíferos en diferentes hábitats, según las dos condiciones climáticas del país. Con la finalidad de conocer la dinámica del sistema depredador-presa-clima que sirvan como bases para la toma de decisiones en el manejo de roedores plaga en los agroecosistemas.

\section{CONCLUSIÓN}

Tyto alba demuestra preferencia por consumir roedores cricétidos, principalmente $Z$. brevicauda, adicionalmente, la mayor tasa de depredación se evidencia durante la temporada de sequía, lo cual coincide con la época cuando ocurre la mayor superficie sembrada de arroz en Venezuela. Estos dos hechos sugieren a $T$. alba como un agente de control biológico potencial en este cultivo.

\section{REFERENCIAS BIBLIOGRÁFICAS}

Aliaga-Rossel, E; Tarifa, T. 2005. Cavia sp. como principal presa de la lechuza de campanario (T.alba) al final de la estación seca en una zona intervenida al norte del Departamento de la Paz, Bolivia. Ecología en Bolivia. 40 (1): 35-42.

Araujo, R; Molinari, J.1995. Variación individual en la dieta de la lechuza (T.alba) en una localidad de los Andes de Venezuela. Resúmenes III Congreso Latinoamericano de Ecología. Fac. de Ciencias. Universidad de los Andes, Mérida, Estado Mérida.

Bellocq, M; Kravetz, F. 1993. Productividad de la Lechuza de Campanario (Tyto alba) en nidos artificiales en Agrosistemas pampeanos. Hornero 013 (04): 277-282.

Bellocq, M; Kravetz, F. 1994. Feeding strategy and predation of the Barn Owl (Tyto alba) and the Burrowing Owl (Speotyto cunicularia) on the rodent species, sex, and size, in agrosystems of central Argentina. Ecología Austral 4:29-34.

Bontzorlos, V; Peris, S; Vlachos, C; Bakaluodis. D. 2005. Diet ofBarn Owl in the agricultural landscapes of Central Greece. Folia Zool. 54(1-2): 99-110.

Bonvicino, C; Bezerra, A. 2003. Use de regurgitated pellets of barn owl (T.alba) for inventorying small mammals in the Cerrado Central Brazil. Studies on Neotropical Fauna and Environment 38(1):1-5.

Brown, J; Twigg, G.1981. Mammalian prey of the Barn owl (T.alba) on Skomer Island, Pembrokeshire. Notes from the Mammal Society.

Campbell, R; Manuawal, D; Harestad, A. 1987. Food habits of the Common Barn Owl in British Columbia. Can J Zool. 65: 578-586.

Carmona, E; Rivadeneira, M.2006. Food habits of the barn owl Tyto alba in the National Reserve Pampa del Tamarugal, Atacama Desert, North Chile. Journal of Natural History 40(7-8): 473-483.

Debus, S; Olsen, J; Rose, B. 2004. Diet of the barn owl Tyto alba near lake forme in arid south Australia. Corella 28 (2): 40-42.

Delgado, C; Calderón, D. 2007. La dieta de la lechuza común Tyto alba (Tytonidae) en una localidad urbana de Urabá, Colombia. Boletín SAO XVII: 94-97.

Delgado, C; Ramírez, J. 2009. Presas de la lechuza común (Tyto alba) en jardín, Antioquia, Colombia. Disponible en: www.ornitologiacolombiana.org/oc8/Delgado.pdf

Fernández, F; Moreira, D; Ferraro, G; De Santis, L. 2009. Presas consumidas por la lechuza de campanario ( $T$. alba) en la localidad de Olavarría, Buenos Aires: un caso elevado de batracofagia. Nuestras Aves 54: 20-2.

Fuentes, L; Poleo, C; Díaz, L. 2012. Potencial depredación de la lechuza de campanario (Tyto alba Scopoli, 1769) sobre roedores en la Estación Experimental del INIACalabozo, Guárico, Venezuela. Mem Fund La Salle de Cienc Nat. 173-174.

Fulhorst, Ch; Bowen, M; Salas, R; Duno, G; Utrera, A; Ksiazek, T; De Manzione, N; Rico-Hesse, R; Shope, R; Betancourt, A; Godoy, O; Millar, E; Vásquez, C; Peters, C; Tesh, R. 1999. Natural rodent host association of Guanarito and Pirital viruses, (Family Arenaviridae) in Central, Venezuela. Am J Trop Med Hyg. 61(2):325-330.

González, D; Ausset, M; Skewes, O; Figueroa, R. 2004. Variación estacional en el consumo de roedores por la lechuza de campanario (T.alba) en un área suburbana de Chillán, Centro-Sur de Chile. Hornero 19 (2): 61-68. 
González, E; Claramunt, S; Saralegui, A. 1999. Mamíferos hallados en egagrópilas de T.alba (Aves, Strigiformes, Tytonidae) en Bagé, Río Grande Do Sul, Brasil. Iheringia Ser Zool (86):117-120.

Goodman, S; Thorstrom, R. 1998. The Diet of the madagascar red owl (Tyto soumagnei) on the Masoala Peninsula, Madagascar. Willson Bull 110(3): 417-421.

Hafidzi, M; Mohd, N. 2003. Prey selection by barn owls in rice fields in Malaysia. En: Rats, mice and people: rodent biology and management Symposium 3: Behaviour. Singleton, G; Hinds, L; Krebs, Ch; Spratt, D., Editores. ACIAR Monograph No 96. Canberra, Australia. 564 p.

Hernández-Muñoz, A; Mancina, C. 2011. La dieta de la lechuza (Tyto alba) (Aves: Strigiformes) en hábitats naturales y antropogénicos de la región central de Cuba. Revista Mexicana de Biodiversidad 82: $217-$ 226.

ICI (Imperial Chemical Industries Americas Inc. Science Series) 1987. A closer look. Portrait the barn owl. Biological Science Department. Bowling Green State University. Ohio. USA. 8 p.

Kitowski, I. 2013. Winter diet of the barn owl (Tyto alba) and the long-eared owl (Asio otus) in Eastern Poland. North-West J Zool 9 (1): 16-22.

Linares, O. 1998. Mamíferos de Venezuela. Editorial Sociedad Conservacionista Audubon de Venezuela. $691 \mathrm{p}$.

López, Y. 2012. Alimentación de la Lechuza (Tyto alba furcata) en Cuba central: Presas introducidas y autóctonas. Tesis de Diploma, Facultad de Biología, Universidad de la Habana. 84 p.

Lugo, L. 2005. Características edafoclimáticas del Sistema de Riego Río Guárico y potenciales en la producción de arroz bajo riego. Modulo 7 2do Curso Básico de Agrometeorología Operativa. INIA Guárico.

Magrini, L; Facure, K. 2008. Barn owl (Tyto alba) predation on small mammals and its role in the control of hantavirus natural reservoirs in a periurban area in southeastern Brazil. Braz J Biol 68(4): 733-740.
MAT (Ministerio de Agricultura y Tierra). 2005-2007. Estadística sobre producción de arroz, Araure, Portuguesa. [Datos suministrados directamente del computador del Ministerio]. Unidad Estatal de Desarrollo Agrícola. División de Estadísticas.

Mendoza, N. 1990. Depredación de las lechuzas sobre las ratas del arrozal. Tesis de grado. Universidad Central de Venezuela. Fac. de Agronomía. Maracay, estado Aragua. Venezuela. 95 p.

Mendoza, R. 2006. Informe de gestión anual 2005. Instituto Nacional de Investigaciones Agrícolas. Centro de Investigaciones Agropecuarias del Estado Guárico, Calabozo. 43 p.

Mendoza, R. 2007. Informe de gestión anual 2006. Instituto Nacional de Investigaciones Agrícolas. Centro de Investigaciones Agropecuarias del Estado Guárico, Calabozo. 49 p.

Mohamad, S; Goh, N. 1991. The use of barn owl (T.alba) to control rice field rats. An experience in Seberang Perak. Mapps Newsletter 15:2-20.

Mohamed, A; Santhanakrishnan, R. 2012. Diet Composition of the Barn Owl Tyto alba (Aves: Tytonidae) and Spotted Owlet Athene brama (Aves: Strigidae) Coexisting in an Urban Environment. Podoces 7(1/2): 21-32.

Mohd, S. 1990. Barn owl (Tyto alba) for controlling rice field rats. Mapps Newsletter 14: 4-51.

Nadeem, M; Khalid, S; Mahmood, T; Kayani, A; Shah, S. 2012. A comparative study of the diets of barn owl (Tyto alba) and spotted owlet (Athene brama) inhabiting Ahmadpur East, Southern Punjab, Pakistan. Anim Biol 62:13-28.

Olsen, P; Olsen, J.1992. Does rain hamper hunting by breeding raptors? EMU 92: 184-187.

Pardiñas, U; Teta, P; Heinonen, S. 2005. Vertebrate prey of the Barn Owl (T.alba) in Subtropical Wetlands of Northeastern Argentina and Eastern Paraguay. J Raptor Res 39 (1): 65-69.

Paredes, H. 2012. Fiebre Hemorrágica Venezolana Fiebre de Guanarito. Botica 9: 1-5. 
Poleo, C; Agüero, D. 2000. Efecto depredador de la lechuza de campanario (T.alba) sobre poblaciones de ratas causantes de daños en el cultivo de arroz. Investigación Agrícola 5: 1-10.

Poleo, C; Garbi, J; Pérez, J. 1998. Lechuza de campanario (T. alba) en el control de roedores en el cultivo del arroz. Fondo Nacional de Investigaciones Agropecuarias. Centro de Investigaciones Agropecuarias del Estado Guárico. 30 p. (Serie $\left.B N^{\circ} 28\right)$.

Poleo, C; Navas, R; Pérez, R; Mendoza, R; Pereira, F. 2011. Especies de plantas observadas en áreas del INIA Guárico Planta Sede. Tríptico.

Ramírez, O; Béarez, P; Arana, M. 2000. Observaciones sobre la dieta de la lechuza de los campanarios en la Quebrada Los Burros (Dpto. Tacna, Perú). Bull Inst Fr Etudes Andin 29 (2): 233-240.

Rocha, R; Ferreira, E; Leite, Y; Fonseca, C; Costa, L. 2011. Small mammals in the diet of Barn owls, Tyto alba (Aves: Strigiformes) along the mid-Araguaia River in central Brazil. Zoología 28 (6): 709-716.

Rubolini, D; Pirovano, A; Borghi, S. 2003. Influence of seasonality, temperature and rainfall on the winter diet of the long-eared owl, Asio otus. Folia Zool 52: 67-76.

Santos-Moreno, A; Alfaro, A. 2009. Mammalian prey of barn owl (Tyto alba) in Southeastern Oaxaca, México. Acta Zool Mex (n.s.) 25(1): 143-149.

Senzota, R. 2012. Plains gerbils (Gerbilliscus robusta) as food of the barn owl (Tyto alba) in the Serengeti Plains (Tanzania): thirty-six years later. Tanz J Sci 38 (2): 101-110.

Shehab, A; Al Charabi, S. 2006. Food of the barn owl T.alba in the Yahmool Are, Northern Syria. Turk J Zool 30: 175-79.
Silva, J. 2003. Biodiversidad de ecosistemas: Sabanas. En: Biodiversidad en Venezuela. Aguilera, M; Azócar, A; González, E, Editores. Fundación Polar. Ministerio de Ciencia y Tecnología. Fondo Nacional de Ciencia, Tecnología e Innovación (FONACIT). Primera Edición Tomo II. Caracas: Venezuela. 1072 p.

Soncini, R; Salas, H; Marcus, L. 1985. Alimentación de la lechuza de los campanarios en (Tyto alba) en San Miguel de Tucumán. Historia Natural 5 (7): 49-54.

Teta, P; Contreras, J. 2003. Primeros antecedentes de la dieta de la lechuza de campanario (Tyto alba) en el Departamento de Neembucu Paraguay. Hornero 18 (1): 57- 59.

Trejo, A; Ojeda, V. 2002. Identificación de egagrópilas de aves rapaces en ambientes boscosos y ecotonales del noroeste de la Patagonia Argentina. Ornitol Neotrop 13: 313-317. Disponible en: www.redpav-fpolar.ifo.ve/ danac/volumen 5art2/index.html

Utrera, A; Duno, G; Ellis, B; Salas, R; De Manzione, N; Fulhorst, Ch; Tesh, R; Mills, J. 2000. Small mammals in agricultural areas of the western Llanos of Venezuela: Community structure, habitat associations, and relative densities. J. Mammal 81(2): 536-548.

Vásquez, C; Salas, R; De Manzione, N; Paredes, H; Basile, L; Alarcón, V. 2005. Fiebres hemorrágicas por Hantavirus en Venezuela. VITAE Academia Biomédica Digital 23. Disponible en: http://caibco.ucv.ve

Vivas, A; Roca, R; Weir, E; Gil, K; Gutiérrez, P. 1986. Ritmo de actividad nocturna de Zygodontomys microtinus, Sigmodon alstoni, y Marmosa robinsoni en Masaguaral estado Guárico. Acta Cient Venez 37:456458.

Zuberogoitia, I. 2000. La influencia de los factores meteorológicos sobre el éxito reproductor de la lechuza común. Ardeola 47(1): 49-56. 